\title{
Quality of obstetric care in the sparsely populated sub-arctic area of Norway 2009-2011
}

\author{
Jan Norum ${ }^{1,2,3^{*}}$, Anca Heyd ${ }^{1}$, Bente Hjelseth ${ }^{4}$, Tove Svee ${ }^{1}$, Fred A Mürer ${ }^{4}$, Randi Erlandsen ${ }^{4}$ and Barthold Vonen ${ }^{5}$
}

\begin{abstract}
Background: It is challenging to obtain high quality obstetric care in a sparsely populated area. In the subarctic region of Norway, significant distances, weather conditions and seasonable darkness have called for a decentralized care model. We aimed to explore the quality of this care.

Methods: A retrospective study employing data (2009-11) from the Medical Birth Registry of Norway was initiated. Northern Norwegian and Norwegian figures were compared. Midwife administered maternity units, departments at local and regional specialist hospitals were compared. National registry data on post-caesarean wound infection (2009-2010) was added. Quality of care was measured as rate of multiple pregnancies, caesarean section, post-caesarean wound infection, Apgar score $<7$, birth weight $<2.5$ kilos, perineal rupture, stillbirth, eclampsia, pregnancy induced diabetes and vacuum or forceps assisted delivery. There were 15,586 births in 15 delivery units.
\end{abstract}

Results: Multiple pregnancies were less common in northern Norway (1.3 vs. 1.7\%) ( $P=0.02)$. Less use of vacuum (6.6\% vs. 8.3\%) $(P=0.01)$ and forceps $(0.9 \%$ vs $1.7 \%)(P<0.01)$ assisted delivery was observed. There was no difference with regard to pregnancy induced diabetes, caesarean section, stillbirth, Apgar score $<7$ and eclampsia. A significant difference in birth weight $<2.5$ kilos $(4.7 \%$ vs. $5.0 \%)(P<0.04)$ and perineal rupture grade 3 and $4(1.5 \%$ vs. $2.3 \%)(P<0.02)$ were revealed. The post-caesarean wound infection rate was higher $(10.5 \%$ vs. $7.4 \%)(P<0.01)$.

Conclusion: Northern Norway had an obstetric care of good quality. Birth weight, multiple pregnancies and post-caesarean wound infection rates should be further elucidated.

Keywords: Quality, Obstetrics, Norway, Registry, Decentralized care, Birth weight, Infection

\section{Background}

Northern Norway covers almost half of Norway's land mass and is about two-thirds of the size of the UK. The population is only 470.000 inhabitants, one fourth of whom live in the two main cities (Bodø and Tromsø). About 2,500 people live in the Norwegian arctic, mainly on the Svalbard islands. Significant distances have been a constant challenge to the northern Norwegian specialised health care in terms of quality of care, costs and logistics. The area has a subarctic and arctic climate causing great challenges, especially during winter time.

\footnotetext{
* Correspondence: jan.norum@helse-nord.no

${ }^{1}$ Northern Norway Regional Health Authority trust, N-8038 Bodø, Norway ${ }^{2}$ Institute of Clinical Medicine, Faculty of Health Sciences, University of Troms $\varnothing$, N-9037 Troms $\varnothing$, Norway

Full list of author information is available at the end of the article
}

Cold and rough weather conditions, long distances, seasonable darkness and snow have to be handled.

Maternity care and delivery may be organized differently in sparsely populated areas. In northern Norway, midwife administered maternity units (MAMUs) have been an important part of health care [1,2]. To secure a high quality of care, Norwegian women have been selected to institution/unit of delivery according to risk factors [1,2]. Generally, three levels of care are available. Those at low risk may deliver at MAMUs [2]. Women with intermediate risk are referred to second-level perinatal care units at local hospitals and those with high risk to a regional specialist hospital for delivery. Whereas the MAMUs generally are staffed with midwives and have a general practitioner on duty available in the community, they have no gynaecologist/obstetrician.

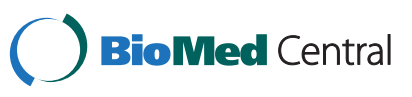


The second level (department of obstetrics and gynaecology) has such a specialist on duty 24 hours a day and emergency caesarean section may be performed at any time. The regional specialist hospitals are superior to the departments at local hospitals in terms of access to paediatricians on duty and a paediatric intensive care unit.

The quality of maternal health care services in Norway has been scrutinised recently. The Ministry of Health and Care Services launched a plan for improved maternity and delivery care entitled 'A Happy Occasion' and incorporated it in the 'Coordination Reform' [3,4]. The main aim of this document is to improve continuous maternity, delivery and postpartum care. The changes to selection criteria and procedures for transportation will result in more centralised care. In this context, in March 2012, we initiated the national registry-based study to analyse the de-centralised obstetric care currently (2009-2011) provided in Northern Norway.

\section{Methods}

Norway has a population of 5 million inhabitants and is divided into four health regions (southeast, western, central and northern region). The northern region has only $9.4 \%$ of the total population and people are scattered within an area of $112,946 \mathrm{~km}^{2}$. To serve the population, the Northern Norway Regional Health Authority (NNR HA) trust runs six midwife managed maternity units (MAMU) (Brønnøysund, Mosjøen, Nordland hospital (NH) Gravdal, Lenvik, Sonjatun, Alta), seven departments of obstetrics and gynaecology (DoOG) [Helgeland hospital (HH) Sandnessjøen, HH Rana, Nordland hospital (NH) Vesterålen, University hospital of north Norway (UNN) Harstad, UNN Narvik, Finnmark hospital (FH) Hammer fest, FH Kirkenes] and two regional specialist hospitals (RSH) (NH Bodø, UNN Tromsø). An overview is shown in Figure 1. The decision concerning level of care is generally carried out early during pregnancy according to national guidelines. Possible occasions are the follow

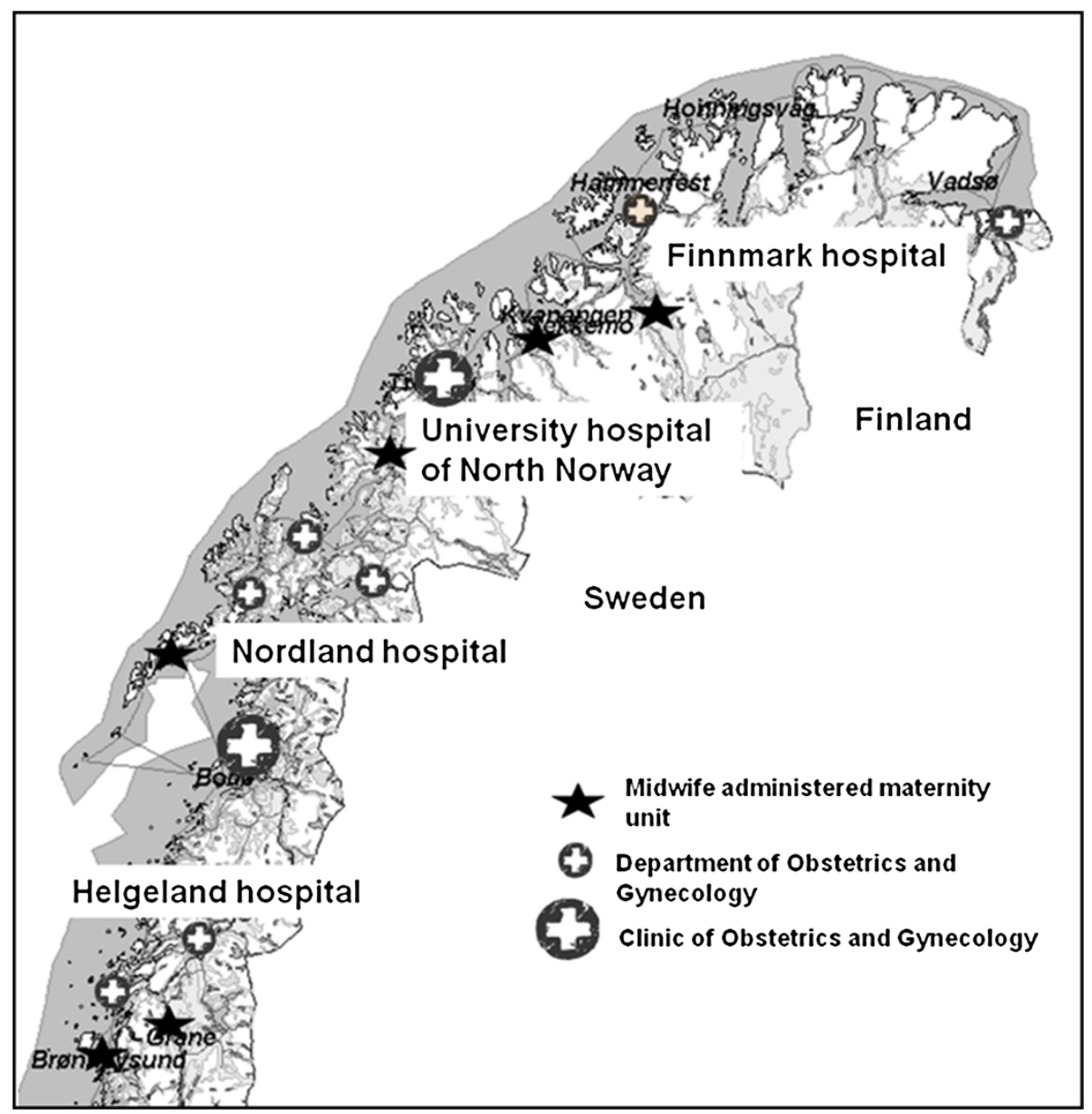

Figure 1 The figure shows the midwife administered maternity units and the departments and clinics of obstetrics and gynaecology and the four hospital trusts in northern Norway. 
up visit in primary health care during the first trimester or at ultrasound screening at $18^{\text {th }}$ week of pregnancy.

\section{Data included}

In Norway, all births are reported to the Medical Birth Registry of Norway (MBRN) (www.fhi.no/mfr). The registry was established in 1967 and was organized in the wake of the thalidomide catastrophe. The particular aim was epidemiological surveillance of birth defects and other perinatal health problems in order to detect, as soon as possible, any future increase in rates. Today it is a national health registry containing information about all births in Norway. The registry aim to clarify the causes and consequences of health problems related to pregnancy and birth, as well as to monitor the incidence of congenital abnormalities. Data for the last years are available online from the MBRN database (www.fhi.no/ $\mathrm{mfr}$ ). In this study, we accessed data (as of March 2012) reported from the 15 institutions in northern Norway and cumulative data from all institutions in Norway. The figures for the three year time period 2009-2011 were analyzed. The following was accessed:

- Births, newborns (only data for 2009-10), born alive or dead, weight below $2.5 \mathrm{~kg}$ and Apgar score $<7$ five minutes after birth, forceps and vacuum assisted delivery $[5,6]$.

- The frequency of multiple pregnancies, pregnancy induced diabetes, eclampsia, perineal rupture grade 3 (partial or total tear through the anal sphincter) or 4 (grade 3 with extension through the rectal mucosa) and caesarean section.

The mentioned outcomes were chosen to describe the quality of care as they were available from the MBRN's online databank (www.fhi.no/mfr). Other factors as birth weight, atonic uterus, bleeding 500-1500 ml, bleeding > $1500 \mathrm{ml}$ and transfusion are parameters registered by the institutions. Unfortunately, these data were, as mentioned, not available from the online databank and consequently not included in our study.

The Norwegian Surveillance Program for Infections in Hospitals (NOIS) (www.fhi.no/nois) was established in 2005. In this registry, surgical wound infections within 30 days after caesarean section are registered prospectively annually from Sept. 1 to Nov. 30. A total of 39 hospitals (9 in northern Norway) participated in the national survey program. Women were followed up after surgery by mail and a phone call from specialized nurses. Infection within 30 days after surgery were registered. Superficial infections could be reported by the women themselves, but all other infections had to be confirmed by a medical doctor. We collected these data for the time period 2009-2011.

\section{Quality control, statistical analysis and authorisation}

Individual data were recorded and analyzed by the MBRN. The quality assurance of the primary data, included linking to the National Population Register (NPR), was performed to identify and confirm the women and collect available information about date of birth and death (in case of death). Furthermore, the reporting institutions were requested to supply additional information when needed. We accessed anonymous and aggregated data from this open source. Similarly data were accessed from the NOIS-registry. The aggregated data were imported to a database at the NNRHA. Microsoft Excel 2007 version was used for the final database, calculations and statistical analysis. The comparison between institutions with regard to quality of care figures was based on rates. Descriptive statistics and the t-test were used for the comparison between institutions. Significance was set to $5 \%$. The t-test was carried out two-sided. Data from the MBRN was available on the Web free of cost and as we imported only aggregated data, no ethical committee or Data Inspectorate approval was necessary. Consequently no approval from the Regional Committees for Medical and Health Research Ethics (REK) was necessary. Similarly, no approval from the Norwegian Social Science Data Services (NSD) was required.

\section{Results}

During the study period there were in total 15,586 births in northern Norway. This accounted for $8.6 \%$ of all births in Norway. Multiple pregnancies were significantly less common $(\mathrm{P}=0.02)$ in northern Norway. The mean percentage was $1.3 \%$ (range $1.2-1.5 \%$ ) and $1.7 \%$ (range $1.7-1.8 \%$ ) in the northern region and Norway, respectively. No twins were born in midwife administered maternity units in northern Norway. The annual figures (20092011) at the 15 northern maternity units are shown in Figure 2. There were 1,332 births $(8.5 \%)$ at MAMUs, 6,712 births (43.1\%) at DoOGs and 7,539 births (48.4\%) at RHSCOGs during study period.

The frequency (all Robson groups) of caesarean section was $16.4 \%$ in northern Norway (Norway 16.7\%) $(P=0.72$ ). The range within the region is shown in Figure 3. Except for NH-Lofoten, no MAMU had any caesarean section performed. [Due to distance to nearest DoOG and rough weather conditions, especially during winter times, $\mathrm{NH}$ Lofoten has a gynaecologist on duty who may perform caesarean section]. Somewhat surprising, the highest frequency of caesarean section was not observed in the regional specialist hospitals, but in the Finnmark hospital (FH) (FH Hammerfest 19.4\% and FH Kirkenes 18.9\%). However, the difference did not reach statistical significance $(P=0.10)$ and the percentage in the Finnmark hospital trust (three locations: Alta, Kirkenes and Ham merfest) was only $16.9 \%$. 


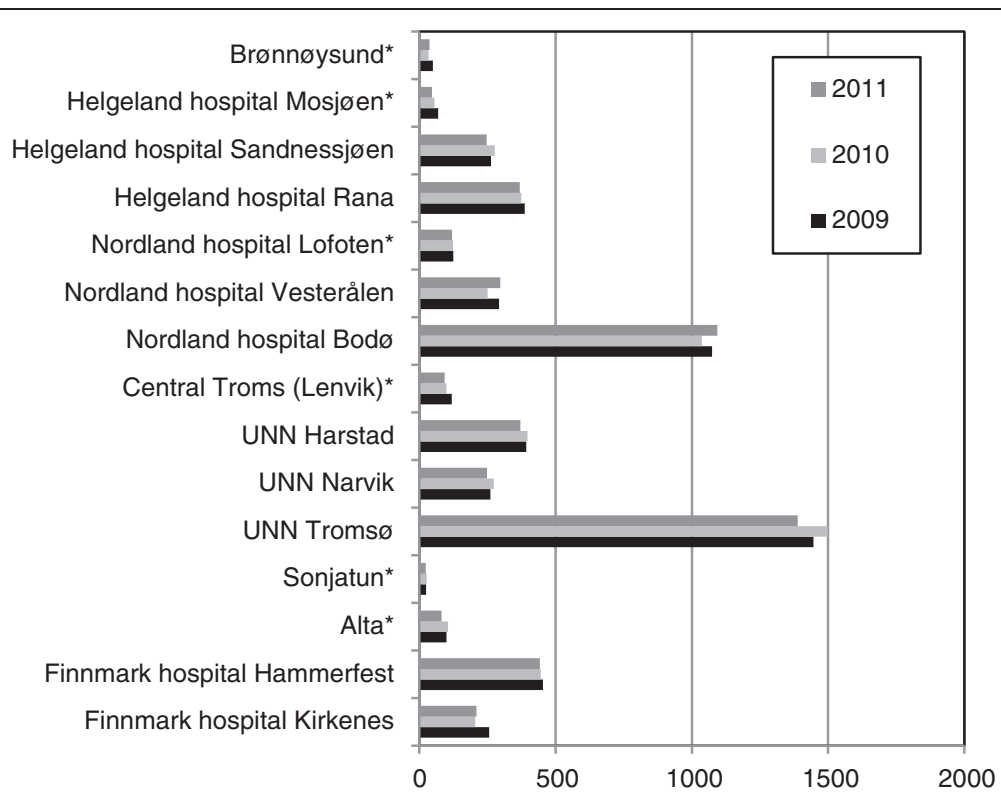

Figure 2 The figure shows the number of deliveries in 2009-2011 according to the 15 delivery units in northern Norway.

The use of forceps delivery was significantly lower in northern Norway than the national figure. Whereas it was employed in $0.3 \%$ of births in our region, the Norwegian figure was almost six times higher $(1.7 \%)(P<0.01)$. None of the northern institutions reached the national level (highest $\mathrm{NH}$ Vesterålen $0.8 \%$ ). Vacuum assisted delivery was also less common in our region $(6.4 \%$ versus $8.3 \%)$ $(P=0.01)$. Only one of the northern institutions reached the national level. Details are shown in Table 1.

Apgar scores below 7 are considered fairly low (critically low $<4$ ). The percentage of Apgar score $<7$ (measured five minutes after birth) was similar (2.1 versus $1.8 \%)$ to the national figure $(P=0.19)$. Due to selection, the three hospitals (FH Hammerfest, UNN Tromsø and NH Bodø) with a paediatric unit had consequently more newborns with lower Apgar score than the others in northern Norway $(P<0.01)$. The number of newborns with a birth weight below 2.5 kilo was significantly higher in our region than in Norway (4.7 vs. $5.0 \%)(P<0.04)$. Generally, these babies should be born in regional specialist hospitals or local hospital departments of obstetrics and gynaecology. Consequently, a significant difference was confirmed

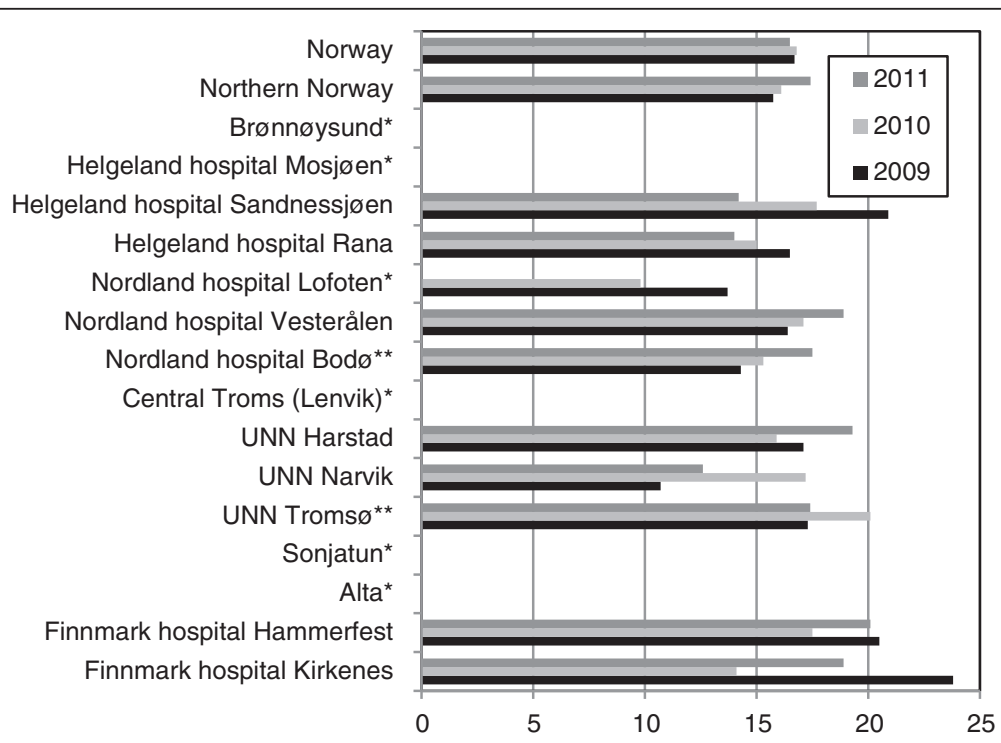

Figure 3 The figure shows the national data and the percentage of caesarean section (all Robson groups) at the various institutions in northern Norway. 
Table 1 Number of births, stillbirths and other birth associated factors in northern Norway and Norway in the time period 2009-2011

\begin{tabular}{|c|c|c|c|c|c|c|c|c|c|c|}
\hline Institution & Births & $\begin{array}{l}\text { Caesarean } \\
(\%)\end{array}$ & $\begin{array}{l}\text { Vacuum } \\
\text { assisted (\%) }\end{array}$ & $\begin{array}{l}\text { Forceps } \\
\text { delivery (\%) }\end{array}$ & $\begin{array}{l}\text { Apgar } \\
\text { score } \\
<7(\%)\end{array}$ & $\begin{array}{l}\text { Weight } \\
<2500 \text { g (\%) }\end{array}$ & $\begin{array}{l}\text { Still-birth } \\
\text { per } 1000\end{array}$ & $\begin{array}{l}\text { Perineal } \\
\text { rupture } \\
\text { G 3-4 (\%) }\end{array}$ & $\begin{array}{l}\text { Eclampsia } \\
\text { per } 1000\end{array}$ & $\begin{array}{l}\text { Pregancy induced } \\
\text { diabetes per } 1000\end{array}$ \\
\hline FH Kirkenes & 671 & 18,9 & 8,1 & 0,5 & 0,9 & 2,4 & 1,6 & 1,2 & 0,0 & 9,1 \\
\hline $\begin{array}{l}\text { FH } \\
\text { Hammerfest }\end{array}$ & 1342 & 19,4 & 5,5 & 1,5 & 2,7 & 4,2 & 2,8 & 1,8 & 1,4 & 21,5 \\
\hline Alta* & 286 & 0,0 & 0,0 & 0,3 & 1,1 & 0,7 & 0,0 & 0,7 & 0,0 & 11,6 \\
\hline Sonjatun* & 76 & 0,0 & 0,0 & 0,0 & 1,4 & 1,4 & 0,0 & 1,3 & 0,0 & 26,2 \\
\hline UNN Troms $\varnothing$ & 4334 & 18,3 & 5,2 & 1,0 & 2,9 & 7,0 & 3,3 & 1,6 & 0,5 & 15,5 \\
\hline UNN Narvik & 782 & 13,5 & 6,0 & 0,4 & 1,1 & 1,5 & 3,6 & 1,1 & 2,4 & 13,5 \\
\hline UNN Harstad & 1160 & 17,4 & 6,7 & 1,0 & 1,2 & 2,4 & 2,5 & 2,0 & 0,8 & 12,3 \\
\hline Lenvik* & 312 & 0,0 & 0,0 & 0,3 & 1,0 & 0,7 & 0,0 & 1,6 & 0,0 & 0,0 \\
\hline $\mathrm{NH}$ Bodø & 3205 & 15,7 & 8,9 & 1,0 & 1,7 & 7,5 & 4,3 & 1,9 & 0,8 & 28,6 \\
\hline NH Vesterålen & 841 & 17,5 & 4,4 & 1,1 & 1,8 & 2,5 & 4,5 & 2,3 & 0,0 & 10,6 \\
\hline NH Lofoten* & 367 & 11,8 & 2,7 & 0,5 & 1,4 & 1,9 & 0,0 & 3,4 & 0,0 & 8,2 \\
\hline HH Rana & 1129 & 15,2 & 8,0 & 1,0 & 1,8 & 1,9 & 2,6 & 2,4 & 0,9 & 9,1 \\
\hline $\begin{array}{l}\mathrm{HH} \\
\text { Sandnessjøen }\end{array}$ & 787 & 17,6 & 7,7 & 0,8 & 2,5 & 2,2 & 6,1 & 1,4 & 2,4 & 14,4 \\
\hline HH Mosjøen* & 170 & 0,0 & 0,0 & 0,0 & 0,0 & 0,5 & 0,0 & 0,6 & 0,0 & 14,8 \\
\hline Brønnøysund* & 121 & 0,0 & 0,0 & 0,0 & 0,0 & 0,0 & 0,0 & 0,0 & 0,0 & 0,0 \\
\hline $\begin{array}{l}\text { Northern } \\
\text { Norway }\end{array}$ & 15586 & 16,4 & 6,6 & 0,9 & 2,1 & 4,7 & 3,4 & 1,5 & 1,1 & 17,4 \\
\hline Norway & 180829 & 16,7 & 8,3 & 1,7 & 1,8 & 5,0 & 3,4 & 2,3 & 0,7 & 17,5 \\
\hline
\end{tabular}

$F H=$ Finnmark hospital, UNN = University hospital of North Norway, $N H=$ Nordland hospital, $H H=$ Helgeland hospital. ${ }^{*}=$ Midwife administered maternity unit.

comparing these units and the MAMUs $(P<0.02)$. Together with the fact that no twins were born in MAMUs and hospitals with a paediatric unit had significantly more newborns with lower Apgar score, this strongly indicated that the selection process was efficient. Details are shown in Table 1.

The stillbirth rate per 1,000 births was the same in the northern region as in Norway (3.4 stillbirths/1,000 births $)(P=0.80)$. This may indicate a similar quality of healthcare during pregnancy in northern Norway as in the other parts of the country. There were no differences between RHSCOGs and DoOGs $(\mathrm{P}=0.75)$. Looking at perineal rupture grade 3 and 4 , our region had a lower figure $(1.5 \%$ vs. $2.3 \%)(P<0.02)$ and within the northern region there was no difference between MAMUs and the others $(1.3 \%$ vs. $1.7 \%)(P=0.28)$. All figures are shown in Table 1.

Eclampsia was not more common in northern Norway (1.1 vs. 0.7 per 1,000$)(P=0.12)$ and within the region there was no difference between the RHSCOGs and DoOGs $(P=0.54)$. The highest figure $(2.4 \%$ ) was revealed in two minor hospitals (Narvik and Sandnessjøen), but these figures must be handled with caution due to low numbers. Details are shown in Table 1.
The frequency of pregnancy induced diabetes was similar in our region as in Norway (17.4 vs. 17.5 per 1,000$)$ $(P=0.99)$. Statistical analyses did not detect any significant difference between MAMUs and DoOGs $(P=0.51)$ or between RHSCOGs and DoOGs $(P=0.07)$.

Looking at the incidence of post-caesarean surgical wound infections, the incidence was higher in northern Norway $(10.5 \%$ vs. $7.4 \%)(P<0.01)$. Details are shown in Table 2. The most striking finding was the difference in rates between the two major regional specialist hospitals in the region $(1.5 \%$ - NH Bodø, $17.2 \%$ UNN Tromsø).

\section{Discussion}

We have indicated that women in northern Norway were offered a similar quality of obstetric care as the one offered to Norwegians in general. Selection criteria with regard to place of delivery seemed to work efficiently. However, there were differences. The use of vacuum and forceps assisted delivery was less common in the northern region. Furthermore, there was revealed significantly more infants with low birth weight $(<2.5$ kilo) and a higher incidence of post-caesarean surgical wound infection in the northern region. 
Table 2 Number of infections following caesarean section during September - November in 2009-2011

\begin{tabular}{llll}
\hline Institution & $\begin{array}{l}\text { Number of } \\
\text { caesarean section }\end{array}$ & \multicolumn{2}{c}{ Number of infections } \\
\cline { 3 - 4 } & 35 & No & $\mathbf{( \% )}$ \\
\hline HH Rana & 35 & 13 & $(11.4 \%)$ \\
FH Hammerfest & 71 & 2 & $(18.3 \%)$ \\
FH Kirkenes & 33 & 2 & $(6.1 \%)$ \\
NH Bodø & 133 & 0 & $(1.5 \%)$ \\
NH Lofoten & 7 & 0 & $(0 \%)$ \\
NH Vesterålen & 25 & 2 & $(3.7 \%)$ \\
UNN Harstad & 54 & 3 & $(15.0 \%)$ \\
UNN Narvik & 20 & 35 & $(17.2 \%)$ \\
UNN Tromsø & 203 & 61 & $(10.5 \%)$ \\
Northern Norway & 581 & 489 & $(7.4 \%)$ \\
Norway & 6637 & &
\end{tabular}

This study has both limitations and strengths. As mentioned in the methods section, more outcomes could beneficially been included. Furthermore, we analysed aggregated data both from the MBRN and the NOIS database. Consequently, sub-analyses on specific subgroups could not be performed. We analysed a short time period (2009-2011). Trends could therefore not be looked at. The strength of this study is that it included all birth in Norway and Northern Norway. By law Norwegian medical institutions and doctors must report every birth. This fundament of the MBRN is one of the reasons for their good quality of data. The fact that the MBRN data are reported in annual reports and as easily available online services to institutions and medical doctors may improve quality and strengthen the reporting doctors'/ institutions' ownership and interest in delivering high quality data.

The caesarean section rate was $16.4 \%$. Within Norway, the lowest figure (12\%) was reported from the Western region (Hordaland county) [7]. The national figures have been rising during the last decade (2000-2010) from 13.6\% to $17.1 \%$ [7]. In England, Scotland, Finland, Sweden and Denmark figures have been reported rising from around $4-5 \%$ in 1970 to $20-22 \%$ in 2001 [8]. Today, across Europe figures vary widely from about $14 \%$ in Nordic countries to $40 \%$ in Italy [9]. Avoidance of unnecessary caesarean section has been a quality target and was one among several topics at the International Forum on Quality \& Safety in Healthcare's meeting in Paris last year $[3,10]$. Overuse of caesarean section exposes both the mother and the fetus to unnecessary risks. To counteract the rise, policy related interventions include the requirement for a second obstetric opinion, education of health professionals, patient and community education, clinical audit and feedback mechanism, clinical practice guidelines, quality improvements strategies and financial incentives $[8,10]$. The benefits of patient decision aids in obstetrics have been explored [11]. When aiming for a reduction in caesarean delivery rate, the trends needs to be monitored carefully in order to prevent a shift from planned to emergency caesarean delivery, as the latter has additional delivery-related risk factors [12].

We revealed an increased risk of low birth weight infants in northern Norway. The association between maternal smoking and hypertension and low birth weight infants has been well established [13,14]. Whereas $18.5 \%$ of pregnant women in Norway were reported smokers, the figure in the northern region was 26.9\% (range between counties $25.4 \%$ - 29.0\%) [7]. Looking at hypertension, $52.0 \%$ of the pregnant women in Norway experienced pregnancy induced hypertension in 2010 [7]. The corresponding figure in northern Norway was also $52.0 \%$. Consequently, maternal smoking habits may be the main culprit of low birth weight in northern Norway. However, there was no notable difference in Apgar score $<7$.

In this study, we disclosed a low rate $(1.5 \%)$ of severe perineal rupture in the northern region. Investigators have reported incidence figures of 1-5\% [15,16]. Risk factors for severe perineal tears are maternal age, parity, race, instrument assistance, episiotomy, birth weight and shoulder dystocia [15]. Looking at our data, we had less use of instrument assistance and more children with low birth weight, This could explain the low rate of severe perineal ruptures in our region.

Multiple births from assisted reproductive technology (ART) have been reported accounting for a substantial proportion of twins and triplets and higher order infants [17]. The number of multiple pregnancies in the ARTsetting in Norway reduced from 1999 to 2010 from $26.5 \%$ to $10.1 \%$ [7]. Whereas $8.6 \%$ of all births in Norway occurred in northern Norway, only $3.9 \%$ of infants conceived by IVF were "made" in this region. The low number of infants conceived by IVF in the northern region may be one explanation for why fewer twins were born in this region.

With regards place of birth in Northern Norway, prematurity is in general the major factor causing altered place of delivery. The new updated selection criteria identified that increasing body mass index (BMI), pregnancy induced diabetes mellitus and previous caesarean section were other major factors in determining place of birth [3]. In common with other developed countries, these diseases are a growing problem in Norway [18]. In our analysis pregnancy related diabetes was $17.4 \%$.

Postoperative infection following caesarean section is common and was more frequent in our region. A Nor wegian study from 2009 revealed that one in 12 women (8.3\%) undergoing caesarean section experienced postoperative infection [19]. The risk was significantly higher among women aged above 29 years. Most infections (86\%) 
were disclosed after hospitalization by the primary health care or the women themselves. The figures were similar or better than reports from other European countries [19]. The significantly higher figures in northern Norway should be investigated. The continuous registration (no longer only 3 months/year) was implemented in late 2012 and more robust data will soon be available. The recent improvement project in Baerum Hospital may be of interest in this setting [20]. They managed to reduce the post-caesarean surgical wound infection rate from $17.4 \%$ to $3.1 \%$. The following areas were focused: Preoperative hair removal, suture for skin closing, dressing in the operation room, wound dressing, double gloving, preoperative surgical hand wash and aseptic techniques.

Neonatal mortality or maternal mortality was not monitored in our survey. The Norwegian figure of neonatal mortality was not available at hospital trust level. According to the MBRN (www.mfr.no), the neonatal mortality rate per 1,000 births in the time period 2002-2011 was 2.3 and 2.2 in Norway and northern Norway, respectively. The corresponding late neonatal mortality rate (7-27 days after delivery) figures were 0.5 and 0.6 respectively. Maternal mortality ratio (MMR) has been analyzed in 22 European countries [21]. The overall figures were 6.3 per 100,000 live births (range 0-29.6). The Norwegian figure in this survey was 3.5. This strongly indicated that maternal death is occurring very rare and consequently not a suitable marker to analyze within the short time interval of our study.

The main characteristic of obstetric service in northern Norway was many delivery units serving a small and scattered population. Following the new national guideline stating that there should be at least four positions for obstetricians at each department, allocation of resources should be considered to cover the extra cost [3]. Especially the high number of units in the southern region (Helgeland) should be analysed. A recent Dutch study has shown that longer travel time from the home to a hospital maternity unit was associated with an increased incidence of intrapartum/neonatal mortality and adverse outcome [22]. This may indicate that our decentralized organization model may be preferable. However, it calls for an efficient selection, updated transport protocols and a safe process. An excellent air-ambulance (fixed wing) and helicopter (rotor wing) service may bring the patients to the correct place of delivery, even when there is a short time left to delivery [23]. These resources may also bring with them a midwife or an obstetrician. To meet people's expectations, the NNRHA trust has 11 air ambulance resources [ 6 planes, 2 ambulance helicopters and 3 search and rescue helicopters] scattered (7 locations) within the region. We had no data clarifying the number of deliveries occurring where they were originally planned. However, bad weather conditions may obviously have changed some plans. Most of northern Norway is located above the Arctic Circle and consequently experience seasonable darkness with no sun for up to four months (Svalbard). Cold and rough weather conditions with snow may also influence on the possibility of air-ambulance and helicopter operations due to risk of icing and landing problems [23]. In India, such a study documented that one third of women delivered at other than their planned place of delivery [24].

In this analysis, we concluded that a national registry (MBRN) was a good surveillance tool to monitor quality of care. A similar conclusion has been given by several investigators $[25,26]$. Shapiro-Mendoza and colleagues in Atlanta GA, USA reported the sudden unexpected infant death case registry a method to improve surveillance [25]. The surveillance system may improve researchers and program planners' ability to create prevention strategies and interventions. Consequently, sudden unexpected infant deaths and injury-related infant deaths may be avoided [25]. The Danish National Indicator Project developed and implemented recently a set of national indicators in their registry [26].

Instruments (screening tools and checklists) are mandatory for the surveillance of quality of care. In the struggle for improved patient safety in midwifery care, Martijn and colleagues identified five domains of patient risk [27]. They were organization, communication, patient-related risk factors, clinical management and outcomes. Based on these, they developed a 32 item screening instrument. They concluded the instrument valid and feasible to assess patient safety and may be used for quantitative analysis of patient records and to identify unsafe situations. The Hospital Corporation of America recently presented their obstetric patient safety efforts and results [28]. Standardization and documentation of critical processes, establishment of national quality benchmarks, reduction in elective deliveries before 39 weeks gestation and reduction in fatal post-caesarean pulmonary embolism were the major areas of progress. A safety culture in the maternity units is important. Raftopoulos and colleagues investigated this in Cyprus [29]. A safety attitude questionnaire was employed and 140 midwives were included in the study. They revealed the highest mean scores on team work and safety climate among the more experienced group of midwives. This could indicate that younger midwives should be focused when efforts are made to improve safety culture.

Today, several European countries run patient safety programs. In Norway, a patient safety campaign called "in safe hands" has been launched. Safe surgery and fewer infections are among the goals. In the UK, Institute for Healthcare Improvement (IHI) has been an important organisation in this setting. Their annual conference has been an important tool in obtaining a focus on quality in maternity care among health care workers 
and administrators. In example, the IHI Scottish Patient Safety Programme (SPSP) recently implemented their Maternity Care Quality Improvement Collaborative (McQIC). (http://www.scottishpatientsafetyprogramme.scot.nhs.uk/ programme/events/McQIC). Hopefully, patient safety culture and focus will reach all European delivery units and further improve the quality of care.

\section{Conclusion}

The Northern Norway Regional Health Authority provides good quality obstetric health care. The differences in birth weight, multiple pregnancies, post-caesarean section wound infections and severe perinatal rupture between Northern Norway and Norwegian should be further elucidated and monitored in the future. Common selection criteria and a national registry are important issues when quality of obstetric care and safety are focused.

\section{Abbreviations}

RSH: Regional specialist hospital; DoOG: Department of obstetrics and gynaecology; IHI: Institute of healthcare improvement; MAMU: Midwife administered maternity unit; MBRN: Medical birth registry of Norway; NNRHA: Northern Norway Regional Health Authority.

\section{Competing interests}

The authors declare that they have no competing interests.

\section{Authors' contributions}

$J \mathrm{~N}, \mathrm{AH}$ and TS initiated the study and designed the protocol. BH, JN, AH, TS, FM and RE participated in acquisition of data. The final database was made by JN and TS. Statistical analysis was done by JN. Suggested sub-analyses, interpretation of data and comments with regard to the results were given by all authors. All study participants have participated in the writing process of the manuscripts and given their approval of the final version.

\section{Acknowledgements}

The authors are thankful to the Medical Birth Registry of Norway who offered data available online on their Website. We also acknowledge the support from the library at the University of Troms $\varnothing$ and the access to necessary infrastructure at the Northern Norway Regional Health Authority trust.

\section{Author details}

${ }^{1}$ Northern Norway Regional Health Authority trust, N-8038 Bodø, Norway. ${ }^{2}$ Institute of Clinical Medicine, Faculty of Health Sciences, University of Tromsø, N-9037 Tromsø, Norway. ${ }^{3}$ Department of Oncology, University Hospital of North Norway (UNN), N-9038 Tromsø, Norway. ${ }^{4}$ Helgeland hospital trust, N-8600 Mo i Rana, Norway. ${ }^{5}$ Nordland hospital trust, N-8092 Bodø, Norway.

Received: 27 November 2012 Accepted: 11 September 2013 Published: 14 September 2013

\section{References}

1. Nesheim Bl, Eskild A, Gjessing L: Does allocation of low risk parturient women to a separate metrinity unit decrease the risk of emergency cesarean section? Acta Obstet Gynecol Scand 2010, 89:813-816.

2. Holt J, Vold IN, Johansen MV, Oian P: Child birth in a modified midwife managed unit: selection and transfer according to intended place of delivery. Acta Obstet Scand 2001, 80:206-12.

3. Ministry of Health and Care Services: Stortinget, report number 12, 2008-9. A happy occasion. A continuous maternity, delivery and postpartum care. Oslo: Ministry of Health and Care Services; 2009.
4. Ministry of Health and Care Services: Stortinget, report number 47, 2008-9. The coordination reform. Correct treatment - at the right placeat the correct time. Oslo: Ministry of Health and Care Services; 2009.

5. Finster $M$, Wood $M$ : The Apgar score has survived the test of time. Anesthesiology 2005, 102:855-857.

6. Apgar $\mathrm{V}$ : A proposal for a new method of evaluation of the newborn infant. Curr Res Anesth Analg 1953, 32:260-267.

7. Norwegian Institute of Public Health: Annual tables for medical birth registry 2010. Births in Norway. Oslo: Norwegian Institute of Public Health; 2012.

8. Khunpradit S, Tavender S, Lumbiganon P, Laopaiboon M, Wasiak J, Gruen $\mathrm{RL}$ : Non-clinical interventions for reducing unnecessary caesarean section. Cochrane Database of Systematic Reviews 2011(6):Art.no. CD005528. DOl 10.1002/14651858.CD005528.pub2.

9. British Broadcasting Cooperation (BBC): Women can choose caesarean birth. BBC; 2011. http://www.bbc.co.uk/news/health-15840743.

10. Mohammade S, Källestål C, Essen B: Clinical audits. A practical strategy for reducing caesarean section rates in a general hospital in Theran, Iran. J Reprod Med 2012, 57:43-48.

11. Say R, Robson S, Thomson R: Helping pregnant women make better decisions. A systematic review of the benefits of patient decision aids in obstetrics. BMJ Open 2011, 1:e000261.

12. Skjeldestad FE, Øian P: Blood loss after caesarean delivery. A registry based study in Norway 1999-2008. Am J Obstet Gynecol 2012, 206(76):e1-7.

13. Murin S, Rafii R, Bilello K: Smoking and smoking cessation in pregnancy. Clin Chest Med 2011, 32:75-91.

14. Czeizel AE, Bánhidy F: Chronic hypertension in pregnancy. Curr Opin Obstet Gynecol 2011, 23:76-81.

15. Baghurst PA: The case for retaining severe perineal tears as an indicator of the quality of obstetric care. Aust N Z J Obstet Gynaecol 2012. doi: 10.1111/ajo.12014.

16. Andersson C, Flems C, Gradel K: Dansk Kvalitetsdatabase for Fødsler. Ugeskr Læger 2012, 174:2547.

17. Sunderam S, Kissin DM, Flowers L, Anderson JE, Folger SG, Jamieson DJ, Barfield WD: Assisted reproductive technology surveillance - United States, 2009. MMWR Surveill Summ 2012, 61:1-23.

18. Ronneberg AK, Nilsson K: Interventions during pregnancy to reduce excessive gestational weight gain: a systematic review assessing current clinical evidence using the grading of recommendations, assessment, development and evaluation (GRADE) system. BJOG 2010, 117:1327-1334.

19. Eriksen $H$, Sæther AR, Løwer HL, Vangen S, Hjetland R, Lundmark H, Aavitsland P: Infections after caesarean section. J Norw Med Assoc 2009, 129:618-622.

20. Dyrkorn OA, Kristoffersen M, Walberh M: Reducing post-caesarean surgical wound infection rate. An improvement project in a Norwegain maternity clinic. BMJ Qual Saf 2012, 21:206-210.

21. Bouvier-Colle MH, Mohangoo AD, Gissler M, Novak-Antolic Z, Vutuc C, Szamotulska K, Zeitlin J: What about the mothers? An analysis of maternal mortality and morbidity in perinatal health surveillance systems in Europe. BJOG 2012, 119:880-890.

22. Ravelli A, Jager K, DeGroot M, Erwich J, Rijninks-van Diel G, Tromp M, Eskes M, Abu-Hanna A, Mol B: Travel time from home to hospital and adverse perinatal outcomes in women at term in The Netherlands. BJOG 2011, 118:457-465.

23. Norum J, Elsbak TM: Air ambulance flights in northern Norway 20022008. Increased number of secondary fixed wing (FW) operations and more use of rotor wing (RW) transports. Int J Emerg Med 2011, 4:55. doi: 10.1186/1865-1380-4-55.

24. Kilaru A, Ganapathy S, Karachiwala B: Quality of care in obstetric services in rural South India. Evidence from two studies with gap of ten years. BMC Proc 2012, 16(Suppl 1):O2. Epub ahead of print.

25. Shapiro-Mendoza CK, Camperlengo LT, Kim SY, Covington T: The sudden unexpected infant death case registry. A method to improve surveillance. Pediatrics 2012, 129:e486-493.

26. Kesmodel US, Jølving LR: Measuring and improving quality in obstetrics the implementation of national indicators in Denmark. Acta Obstet Gynaecol Scand 2011, 90:295-304.

27. Martijn L, Jacobs A, Harmsen M, Maassen I, Wensing M: Patient safety in midwifery care for low-risk women. Instrument development. J Midwifery Womens Health 2012, 00: 1-10. doi:10.1111/j.1542-2011.00147. 
28. Clark SL, Meyers JA, Frye DK, Perlin JA: Patient safety in obstetrics - the hospital corporation of America experience. Am J Obstet Gynecol 2011, 204:283-287.

29. Raftopoulos V, Savva N, Papadopoulou M: Safety culture in the maternity units. A census survey using the safety attitudes questionnaire. BMC Health Serv Res 2011, 11:238.

doi:10.1186/1471-2393-13-175

Cite this article as: Norum et al:: Quality of obstetric care in the sparsely populated sub-arctic area of Norway 2009-2011. BMC Pregnancy and Childbirth 2013 13:175.

\section{Submit your next manuscript to BioMed Central and take full advantage of:}

- Convenient online submission

- Thorough peer review

- No space constraints or color figure charges

- Immediate publication on acceptance

- Inclusion in PubMed, CAS, Scopus and Google Scholar

- Research which is freely available for redistribution 